INDEX FOR VOLUME 6, 2015

\title{
HEAT PIPE SCIENCE AND TECHNOLOGY
}

\author{
TABLE OF CONTENTS FOR VOLUME 6, 2015
}

PAGE NUMBERS FOR ISSUES:

Issue 1-2: 1-120; Issues 3-4: 121-270

\section{ISSUES 1-2}

Structural, Thermophysical, and Mechanical Characteristics of Metal Felt Wicks of Modern Heat Pipes

A. Gershuni, V. Zaripov, \& V. Baturkin

Comparative Analysis of Heat-Transfer Capability of Evaporation-Condensation and Recuperative Tubular Heat Exchangers

A. Gershuni, A. Nishchik, \& V. Razumovskiy

Investigation of Loop Heat Pipe Oscillating Behavior using Numerical Simulation D. Nesterov \& G. Dmitriev

Accurate Temperature Measurements of a Heat Pipe with Pressure Control:

Design Descriptions and Test Results

S. Chen, Z. Xue, D. Deng, \& W. Qu

Experimental Study on Pulsating Heat Pipe using Self-Rewetting Fluid as a Working Fluid: Visualization of Thin Liquid Film and Surface Wave

K. Fumoto, T. Ishida, T. Kawanami, \& T. Inamura

Experimental Study of Electron Beam Irradiation on an Oscillating Heat Pipe N. Iwata, H. Ogawa, Y. Miyazaki, \& S. Fukuda

Pulsating Heat Pipe in Hypergravity Conditions M. Mameli, M. Manzoni, L. Araneo, S. Filippeschi, \& M. Marengo

Development of Miniature Heat Switch-Temperature Controller Based on a Variable Conductance Loop Heat Pipe

D. Mishkinis, J. Corrochano,\& A. Torres

\section{ISSUES 3-4}

Preface: 9th Minsk International Seminar

L.L. Vasiliev \& A. Zhuravlyov

Experimental Characterization of a Double Back to Back Pulsating Heat Pipe for Power Electronics

B. Agostini \& M. Habert

Microreactors for Biodiesel Synthesis: Design, Fabrication, and Characterization C.P. Naveira-Cotta, C.P. Tostado, J.M. Costa, \& J.S. Nunes 
Effectiveness of Combined Subcooler-Capillary Blocker Device in LHP for Space Applications

Anatoly P. Lukisha \& Donatas Mishkinis

Copper-Water Loop Heat Pipes: Issues and Achievements

Yu. Maydanik \& V. Pastukhov

A Study on Phenol Resin-Based Adsorbents + Ethanol Pairs for Adsorptive

Cooling/Refrigeration

B.B. Saha, I.I. El-Sharkawy, \& S. Koyama

Effects of Fin Spacing and Fin Height of Capillary-Assisted Tubes on the Performance of a Low Operating Pressure Evaporator for an Adsorption Cooling System

P.C. Thimmaiah, A. Sharafian, W. Huttema, \& M. Bahrami

Experimental Investigations of Aluminum Thermosyphons for a Photovoltaic Thermal Module

S. Khairnasov, B. Rassamakin, \& D. Kozak

Development of Multilayer Porous Media using Colloidal Processing

S.T.R. Velásquez, G.G.V. Nuernberg, J.P.M. Florez, L.E. Vieira, M.B.H. Mantelli, \& A.N. Klein

Evaluation of CaCl2-Silica Gel Sorbent for Water Sorbtion Cooling Systems

C. McCague, K. Fayazmanesh, C. Berlanga, M. Bahrami

Thermodynamic Modelling of Thermoelectric Generator Systems

S. C. Kaushik, S. Manikandan, R. Hans

Index to Volume 6 
INDEX FOR VOLUME 6, 2015

\section{HEAT PIPE SCIENCE AND TECHNOLOGY}

\section{AUTHOR INDEX FOR VOLUME 6, 2015}

PAGE NUMBERS FOR ISSUES:

Issue 1-2: 1-120; Issues 3-4: 121-270

\author{
Agostini, B., 123 \\ Araneo, L., 91 \\ Bahrami, M., 195, 229 \\ Baturkin, V., 1 \\ Berlanga, C., 229 \\ Chen, S., 51 \\ Corrochano, J., 111 \\ Costa, J.M. Jr., 135 \\ Deng, D., 51 \\ Dmitriev, G., 25 \\ El-Sharkawy, I.I., 183 \\ Fayazmanesh, K., 229 \\ Filippeschi, S., 91 \\ Florez, J.P.M., 217 \\ Fukuda, S., 77 \\ Fumoto, K., 65 \\ Gershuni, A., 1, 11 \\ Habert, M., 123 \\ Hans, R., 241 \\ Huttema, W., 195
}

Inamura, T., 65

Ishida, T., 65

Iwata, N., 77

Kaushik, S.C., 241

Kawanami, T., 65

Khairnasov, S., 205

Klein, A.N., 217

Koyama, S., 183

Kozak, D., 205

Lukisha, A.P., 155

Mameli, M., 91

Manikandan, S., 241

Mantelli, M.B.H., 217

Manzoni, M., 91

Marengo, M., 91

Maydanik, Yu., 167

McCague, C., 229

Mishkinis, D., 111, 155

Miyazaki, Y., 77

Naveira-Cotta, C.P., 135
Nesterov, D., 25

Nishchik, A., 11

Nuernberg, G.G.V., 217

Nunes, J.S., 135

Ogawa, H., 77

Pastukhov, V., 167

Qu, W., 51

Rassamakin, B., 205

Razumovskiy, V., 11

Saha, B.B., 183

Sharafian, A., 195

Thimmaiah, P.C., 195

Torres, A., 111

Tostado, C.P., 135

Velásquez, S.T.R., 217

Vieira, L.E., 217

Xue, Z., 51

Zaripov, V., 1 


\section{INDEX FOR VOLUME 6, 2015}

\section{HEAT PIPE SCIENCE AND TECHNOLOGY}

\section{SUBJECT INDEX FOR VOLUME 6, 2015}

PAGE NUMBERS FOR ISSUES:

Issue 1-2: 1-120; Issues 3-4: 121-270

activated carbon, 183

adsorption cooling system, 195

adsorption, 183

aluminum grooved heat pipe, 205

biodiesel synthesis, 135

building facade integration, 205

calcium chloride, 229

capillary structure, 1

capillary-assisted evaporation, 195

comparison of transferred heat

fluxes, 11

condenser, 167

cooling, 183

copper, 167

effectiveness, 155

efficiency, 205

electron beam irradiation, 77

enhanced tube, 195

ethanol, 183

evaporation-condensation and recuperative tubular heat

exchangers, 11

evaporator, 167

exergy analysis, 241

exergy efficiency, 241 feedback of pressure to temperature, 51

gamma ray, 77

heat pipe, 1, 51

heat transfer, 205

hypergravity, 91

irreversibilities, 241

large-diameter centrifuge, 91

liquid film, 65

loop heat pipe, 25, 155, 167

loop heat pipes, 111

low-operating pressure, 195

maximum heat transfer, 77

metal fiber, 1

microfabrication, 135

microreactors, 135

microscopic characterization, 135

miniaturization, 111

numerical simulation, 25

oscillating heat pipe, 77

photovoltaic thermal module, 205

porosity, 217

power electronics, 123

pulsating heat pipe, 65, 91, 123

radiation in a vacuum as a

boundary condition, 155 reactive flow, 135

self-rewetting fluid, 65

slip casting, 217

solar cells, 205

sorption cooling, 229

space applications, 111

space holder, 217

subcooler-capillary blocker, 155

temperature calibration, 51

temperature measurement, 51

thermal conductance, 77

thermal control, 111

thermal tests, 111

thermally powered, 183

thermoelectric generator, 241

thermosyphon, 205

transient model, 25

two-phase cooling, 123

two-phase flow, 91

two-phase heat transfer

technology, 111

two-way pressure regulating, 111

visualization, 65

water isotherm, 229

water, 167

working fluid, 167 